$\mathrm{U}$ niversity of Oxford researchers have shed light on how perovskites rapidly form macroscopic single crystals at high temperatures from solution. Using this knowledge, they were able to produce higher quality single crystals than ones made so far using the rapid crystallization technique.

Henry Snaith and his colleagues report in Nature Communications (doi:10.1038/ ncomms13303) that perovskite crystallization is triggered by a change in the acid-base equilibrium of the solvent, which raises the concentration of the solute and results in the perovskite's quick saturation out of the solution as crystals. Understanding the factors that influence and control crystallization is key to making high-performance perovskite optoelectronic devices. ncreasing the stability of
perovskites is another key requirement for their commercial success. Michael Grätzel and co-workers at the École Polytechnique Fédérale de Lausanne, Switzerland have now made highly stable, efficient solar cells by integrating rubidium into lead-halide perovskite films.
The resulting solar cells have a power-conversion efficiency of $21.6 \%$. They maintain $95 \%$ of their initial performance over 500 continuous hours under full sunlight at $85^{\circ} \mathrm{C}$.

Earlier this year, the researchers reported a triple-cation (methylammonium, formamidinium, and cesium) perovskite, yielding a solar efficiency of $21.2 \%$ that remained stable for 250 hours. The new advance published in Science (doi:10.1126/science. aah5557) takes that work a step further. This time they added rubidium to the mix. The rubidium cations may help relax lattice strain, giving a more defect-free crystal, says the lead author Michael Saliba.
$\mathrm{R}$ esearchers have used perovskite quantum dots in solar cells to get a relatively high power-conversion efficiency of $10.77 \%$. This is comparable to efficiencies of quantum dot solar cells made of other materials, and higher than that of other reported all-inorganic perovskite solar cells.

Quantum dots are nanocrystals of semiconductor materials. The re- searchers, led by Joseph M. Luther at the National Renewable Energy Laboratory, made a thin film of nanocrystals of the perovskite cesium lead iodide $\left(\mathrm{CsPbI}_{3}\right)$ with good electronic coupling among the quantum dots. Low-bandgap all-inorganic perovskites such as $\mathrm{CsPbI}_{3}$ were thought to be stable only at temperatures over $600^{\circ} \mathrm{F}$. But the team discovered a method to keep nanocrystals of the material stable at room temperature, which they detail in Science (doi:10.1126/science. aag2700). They first mixed a Cs-oleate solution with a $\mathrm{PbI}_{2}$ precursor. They then purified the nanocrystals using methyl acetate as an anti-solvent that removed excess unreacted precursors, which turned out to be critical to increasing their stability.

\title{
2016 Nobel Prizes in physics and chemistry: A materials view
}

Prachi Patel

$\mathrm{T}$ his year's Nobel Prizes in Chemistry and Physics, which honor the use of topological concepts, were also a win for materials science and materials research.

The 2016 Nobel Prize in Physics was awarded to David J. Thouless at the University of Washington, F. Duncan M. Haldane at Princeton University, and J. Michael Kosterlitz at Brown University for their explanations of exotic states of matter using the mathematical concept of topology. The Nobel Prize in Chemistry went to Jean-Pierre Sauvage at the University of Strasbourg, France; Sir J. Fraser Stoddart at Northwestern University; and Bernard L. Feringa at the University of Groningen, The
Netherlands, for creating the world's smallest machines by synthesizing topologically very challenging structures on the molecular scale.

"This was very joyful to hear because topology is an elegant concept and finally it got recognized," says Avadh Saxena, a physicist at Los Alamos National Laboratory who works in the area of topological concepts in materials science.

Both prizes recognized fundamental research that has led and will continue to fuel materials discoveries. "In a time when funding tends to be skewed toward application-driven research, it's fantastic to see that the Nobel committee has given a nod to basic research with huge amounts of promise," says Alexander Spokoyny regarding the Chemistry Nobel Prize. Spokoyny, a professor of chemistry at the University of California, Los Angeles, studies molecular synthesis.

Topology describes the properties of a material that are preserved under continuous deformation such as stretching. The electrical current through a wire, for instance, remains the same when the wire is deformed, Saxena explains. "Just as geometry is useful for understanding materials, topology is also useful in understanding material behaviors," he says.

The theoretical work that the physics laureates pioneered laid the groundwork for explaining unusual behaviors that 
experimentalists saw in extremely thin layers and at material surfaces. At the University of Birmingham, UK, in the 1970s, Kosterlitz and Thouless showed that topological phase transitions - the shifting of materials between states with different topologies - were possible on materials surfaces or inside extremely thin layers that could be considered twodimensional. Until then it was thought that superconductivity (zero resistance) and superfluidity (zero viscosity), phase transitions that occur in certain materials at very low temperatures, only occurred in fully three-dimensional materials.

Kosterlitz and Thouless used their models to explain the phase transition mechanism that makes superconductivity vanish at high temperatures. Their theory, known as the KT transition, is universal and applies to different materials at surfaces and inside ultrathin layers.

Then in 1982, Thouless used topology to explain the mysterious phenomenon known as the quantum Hall effect. When a thin conducting layer between two semiconductor slabs, cooled to near absolute zero, sits in a strong perpendicular magnetic field, voltage develops across it. The conductivity, strangely, increases stepwise as the magnetic field strength increases.

Around the same time, Haldane was applying topology to chains of magnetic atoms. These atoms have a nucleus of an even or odd mass number depending on whether their quantum property, known as nuclear spin, is a full or half integer. Haldane predicted that certain chains of the atoms have topological properties that result in a half-integer spin at the end. This implies that by controlling topology, researchers could encode information in materials.

The physicists' work has led to breakthroughs in materials research and condensed-matter physics, including the development of topological insulators, topological superconductors, Dirac semi-metals, and Weyl semi-metals, which have been vital for fundamental physics research but could also have engineering applications. For example, topological insulators, which do not conduct electricity in their bulk but do so on

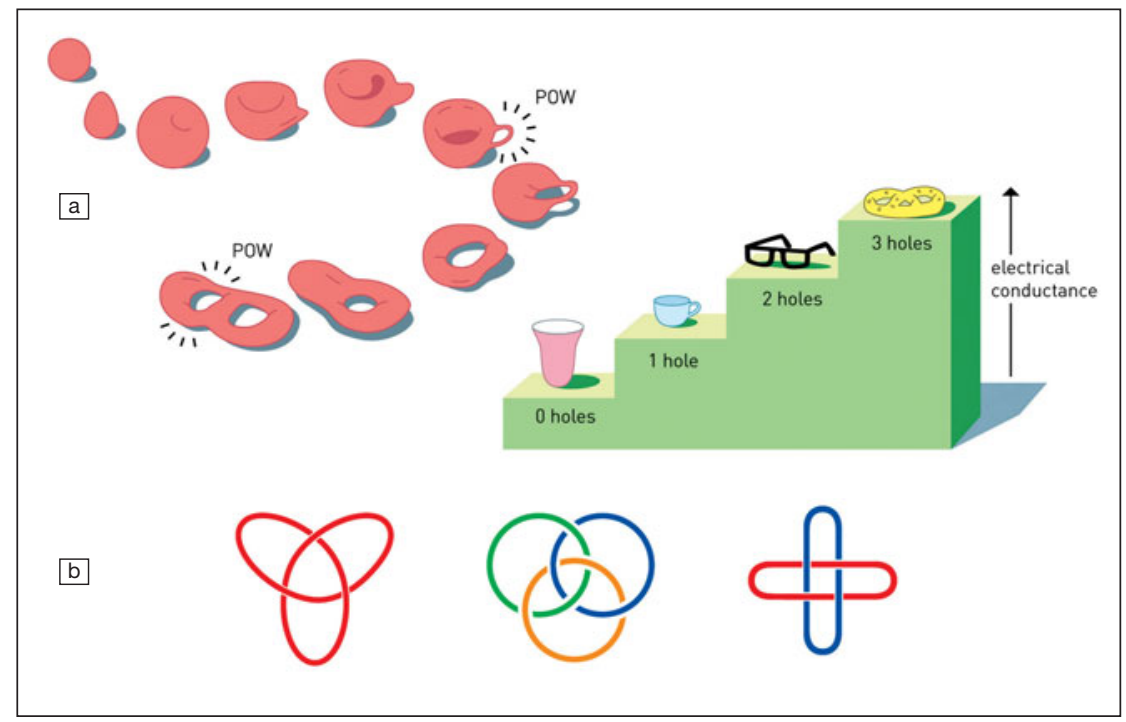

(a) Change in topology of an object when a hole is created (left); distinction between different topological objects characterized by number of holes (i.e., genus) (right). (b) Topology of molecular building blocks used by laureates for creating molecular machines: (left to right) trefoil knot, catenane, and solomon link. Illustration: (C) Johan Jarnestad/Royal Swedish Academy of Sciences.

their surface, "could find use in sensors and high-capacity information storage, but their most exciting application might be quantum computing," Saxena says.

The Chemistry Nobel Prize laureates built molecular-scale mechanical devices that can be controlled with small inputs of energy. The first major breakthrough, involving topological entanglement, came in 1983 when Sauvage discovered a way to interlock ring-shaped molecules. This was the first time molecules were linked mechanically and not through covalent bonds in which atoms share electrons.

Eight years later, Stoddart made a molecule with a ring component that moved along an axle when triggered by heat. Stoddart's group has since used the ring-axle molecules to make an elevator that can move up and down with changes in $\mathrm{pH}$, an artificial muscle in which the molecules bend a gold film, and a computer chip that is like a molecular abacus. Sauvage and Stoddart have gone on to make molecules with very complicated topologies, such as the knots and links seen in Celtic art.

Feringa, meanwhile, built the first molecular motor in 1999: a rotor bladelike molecule that spins in one direction when exposed to ultraviolet light. In
2011, his research group built a fourwheel-drive nanocar, in which a molecular chassis moved on four of the molecular motors acting as wheels.

The chemists' work "reinvigorated the field of topological chemistry," which involves interlocking molecules in complicated structures, the Royal Swedish Academy says. Nature has molecular machines that, for instance, move cellular materials and construct proteins. Artificial molecular machines have a long way to go to catch up, but the laureates' research lit the first spark. "Biological systems are capable of rapidly constructing shape-actuated molecules," Spokoyny says. "Many classes of biological molecules use mechanical bond interactions for assembly. The Nobel laureates were able to achieve that synthetically: rapidly construct mechanical bonds of all sorts on the bench."

Several research groups are now exploring the use of molecular machines for different applications. A simple example of such a machine is a nanovalve, Spokoyny says. Researchers have already demonstrated nanoparticles with light- or redoxactivated valves that could serve as smart drug delivery vehicles, and metal-organic frameworks that open and close pores on demand for energy storage and sensors. 